\title{
Philosophiques
}

Josiane Boulad-Ayoub et François Blanchard, Les grandes figures du monde moderne, Paris, L'Harmattan, 2001, 579 p.

\section{Axelle Martin}

Volume 29, numéro 2, automne 2002

La démocratie délibérative

URI : https://id.erudit.org/iderudit/006314ar

DOI : https://doi.org/10.7202/006314ar

Aller au sommaire du numéro

Éditeur(s)

Société de philosophie du Québec

ISSN

0316-2923 (imprimé)

1492-1391 (numérique)

Découvrir la revue

Citer ce compte rendu

Martin, A. (2002). Compte rendu de [Josiane Boulad-Ayoub et François

Blanchard, Les grandes figures du monde moderne, Paris, L'Harmattan, 2001,

579 p.] Philosophiques, 29(2), 411-418. https://doi.org/10.7202/006314ar d'utilisation que vous pouvez consulter en ligne.

https://apropos.erudit.org/fr/usagers/politique-dutilisation/ 
inclut la poésie. Cette pratique pourrait choquer certains lecteurs puristes qui aiment voir en Platon un écrivain cultivant le goût du mystère et de l'allusion, mais il est par ailleurs certain qu'elle est le résultat d'un louable parti pris de transparence de la part du traducteur. En tout état de cause, elle indique tant aux hellénistes qu'aux autres lecteurs les endroits où ils doivent être vigilants.

Q uant aux notes, elles subissent le même manque d'espace que l'introduction et se réduisent donc à l'essentiel. Pour qu'elles soient aussi complètes que celles des autres éditions de la même collection, qui ajoutent à la traduction un appareil de notes parfois imposant, il aurait fallu en doubler le volume. Compte tenu que cette collection se destine à un large lectorat, il est toutefois concevable qu'elles aient ces proportions sans que cela prête flanc à la critique. G. Leroux leur donne la plus grande utilité en éclairant la traduction par une explication des concepts grecs et en renvoyant aux études les plus profitables sur le sujet. Les liens avec les auteurs classiques et les œuvres de Platon aideront aussi le lecteur peu familier de la littérature grecque à s'y retrouver dans ce texte à l'immense portée intertextuelle. Aux grandes articulations du débat, elles fournissent de plus une explication substantielle de son enjeu et des conséquences des solutions que propose Platon. S'y ajoutent une bibliographie (24 pages), une série de tableaux explicatifs et les index habituels (noms propres, thèmes, et auteurs anciens).

Cette nouvelle République remplit donc entièrement la fonction d'un efficace outil de travail qui tire le meilleur parti d'un espace passablement réduit en offrant une foule de renseignements nécessaires à une compréhension à la fois originale et authentique du chef d'œuvre de Platon.

BEN OÎT CASTELNÉRAC Université de Montréal

\section{Josiane Boulad-Ayoub et François Blanchard, Les grandes figures du monde moderne, Paris, L'H armattan, 2001, 579 p.}

Le collectif publié sous la direction de M adame Boulad-Ayoub et François Blanchard est la publication d'une série d'interventions et de textes produits dans le cadre d'un cours virtuel de la Chaire Unesco-U qam de philosophie. Les textes sont le plus souvent ceux de $\mathrm{M}$ adame Boulad-Ayoub, François Blanchard s'étant chargé plus spécifiquement, mais non exclusivement, des chapitres sur I'histoire des sciences, et les autres textes sont dus à Benoît M ercier, Paul Dumouchel, Sonia Déragon, Benoît Pépin et Jürgen H eizmann.

On nous y propose une promenade à travers une longue période de l'histoire des idées, allant de la Renaissance à la R évolution française. Le choix de ces périodes est justifié par le contenu même du livre : la Renaissance y est présentée comme un moment d'éveil et d'apparition d'une nouvelle vision de l'homme et du monde, et en ce sens comme un point d'origine. La R évolution marque le point d'arrêt de cette période en ce qu'elle représente en quelque sorte l'aboutissement du long chemin de ces idées de l'humain, de la nature, de la religion, à travers quatre siècles au cours desquels s'accomplira le passage de l'humanisme à l'universalisme. Le fil rouge du livre, comme l'indique son titre, sa problématique sous-jacente, est la notion de figure. II s'y passe en effet deux choses :

Les auteurs ont choisi de mettre l'accent sur les hommes, sur la subjectivité des savants, des auteurs, des peintres, au point de vue de la figure, précisément, 


\section{2 · Philosophiques / Automne 2002}

c'est-à-dire de ce qui les rend singulier. On ne nous présente pas des exposés froids et simplement théoriques sur les grands penseurs ou artistes et leurs systèmes, mais un regard humain sur les idées. Le choix des textes en témoigne: qu'ils soient canoniques ou moins connus, dans presque tous, leurs auteurs nous y parlent des crises personnelles qui ont précédé les grandes intuitions, des moments de création, du désir qu'ils ont de connaître, de savoir et de faire savoir ; il y est question de l'esprit, oui, mais jamais du froid concept. Le lecteur peut voir l'esprit incarné. La forme volontairement non didactique du livre (illutrations, encadrés explicatifs, biographiques ou synthétiques) accentue ce côté chaleureux : les hommes dont il est question ici nous deviennent proches, on les suit dans leur aventure spirituelle, on découvre comment ils sont parvenus à leurs systèmes ou leurs positions théoriques. Parallèlement, et puisque le livre traite principalement des personnages connus, il est fait mention des éventuelles légendes ou folklores divers qui circulent dans la vulgate à leur propos, et les auteurs ont soin de les évoquer pour s'en démarquer, sinon par une analyse fouillée qui dépasserait le cadre de l'ouvrage, du moins par des remarques critiques plus nuancées.

$M$ ais nous voyons la légitimation de ce livre et du choix de la période traitée dans le fait, justement, que le point de départ choisi, la R enaissance, est celui de l'émergence de la notion même de figure. Parce que l'homme se recentre sur lui et pense le monde d'une manière nouvelle, à travers sa propre singularité humaine et terrestre, il peut devenir une figure, et c'est pourquoi ce livre peut être lu comme le récit de la naissance même des figures du monde moderne, de leur esquisse jusqu'à leur complet développement.

Les auteurs ne nous livrent pas un exposé détaillé et savant de tout ce qui s'est fait au long de cette vaste période, mais bien plutôt une promenade à travers quelques siècles d'histoire, avec des pauses qui sont comme autant d'arrêts sur image, lorsque nous croisons ces grandes figures qui font l'objet de l'ouvrage. Certains chapitres sont consacrés exclusivement à un auteur (D escartes, Spinoza, Voltaire, Kant, etc.), et présentent, à côté des éléments principaux de sa biographie, l'exposé de ses positions théoriques fondamentales et le contexte socio-historique de leur apparition. L'ampleur de la période traitée empêche naturellement que toutes les personnalités marquantes de ces siècles soient examinées dans le détail, et les auteurs sont parfois dans l'obligation de ne parler d'un philosophe, d'un écrivain, que par allusion, ou en utilisant des raccourcis qu'on peut parfois discuter, mais ils sont tous universitaires et au fait de leurs sujets, et de ce fait évitent toujours l'écueil classique de la «simplification contre-sens».

Le désir qu'ont eu les auteurs de rendre plaisant et agréable ce cheminement parmi les figures essentielles et les événements de ces siècles, est encore attesté par le choix des liens internet proposés dans le CD-R om accompagnant l'ouvrage. C e CDRom contient l'intégralité du texte du livre, auquel est adjoint, par chapitre, une liste de sites internet pour chaque thème croisé. Ces sites sont eux-mêmes une invitation à la découverte, mais non sur le mode érudit : on nous propose, ici un texte de $M$ achiavel, là une vie de Shakespeare ou ses poèmes, ailleurs une mise au point sur I'histoire des religions, sur le règne de Louis XIV.... Des morceaux choisis du côté du cœur, dirons-nous, par petites touches, et qui dessinent pour le lecteur un paysage intellectuel riche et varié dans lequel il aura plaisir à se perdre. Et pour ceux qui désirent poursuivre leurs investigations de manière plus savante, chaque site, comme il est 
d'usage sur internet, donne accès à une autre série de liens, le lecteur ayant ainsi la possibilité de retrouver des sites aux formes plus académiques ou scientifiques (on regrettera seulement qu'un certains nombre de liens cités, en particulier ceux du serveur Géocities, soient désormais inaccessibles).

L'ouvrage contient trois axes, trois périodes historiques : les siècles de l'aventure, couvrant la période de la Renaissance, et identifiés à un moment d'éveil, au cours duquel I'homme se recentre sur lui-même et reconsidère parallèlement sa conception de la nature et de l'univers; le siècle de la raison, le XVII ${ }^{\mathrm{e}}$ siècle, avec l'émergence de la pensée rationnelle, le développement de l'instrument raison pour régler les questions politiques, esthétiques, spéculatives. On trouve dans cette partie un exposé des positions rationalistes et empiristes. Enfin le siècle des Lumières, le combat qui y est mené contre l'obscurantisme et le despotisme, l'expansion du savoir de manière cosmopolite, et l'aboutissement de ces grands mouvements intellectuels et sociaux dans la Révolution Française.

Dans la première partie, consacrée aux siècles de la Renaissance, les auteurs montrent comment vont se mettre en place les éléments qui constituent des points de départ au voyage dans lequel on veut nous emmener. Les auteurs font un tour d'horizon, assez complet, si l'on considère la richesse intellectuelle et artistique de cette époque, de ce qui se produit dans le domaine des arts, des lettres, et des sciences, de la religion, en mettant l'accent sur le caractère fondateur et novateur de ces produits de l'esprit.

On y montre ainsi comment la R enaissance est le moment où l'homme, se séparant du ciel sans pourtant en nier l'existence ou l'importance, va se focaliser sur luimême, et acquérir par là une figure (ce qui justifie le titre del'ouvrage), comment il en vient à se prendre pour objet de réflexion et d'attention. C'est le moment de I'H umanisme, qui, donnant une nouvelle perspective sur I'homme terrestre et sa nature, va préparer le terrain pour la mise en place de la problématique du sujet, qui s'élaborera dans les siècles suivants. On découvre par exemple les débuts de la notion de propriété intellectuelle, et ses origines.

II est montré parallèlement comment cet homme renaissant va rendre le monde commensurable à ses mesures et le considérer d'un œil nouveau (la perspective et la construction de l'espace en peinture, dans un chapitre sur l'art). Les sciences prennent alors un tour nouveau, l'astronomie, les mathématiques, la science du vivant, tout en s'élevant à un plus grand niveau d'abstraction et de précision, cherchent cependant à penser le réel (la navigation, les finances, la chirurgie etc.).

Ce qui apparaît dans la présentation qui est faite des événements intellectuels de cette période, c'est une sorte de focalisation sur un monde désormais mesuré, calculable, rationalisé, dirons-nous, une nouvelle conception de la réalité elle-même. Le rapport de l'homme à Dieu est réinterrogé, c'est le moment des réformes et des contres-réformes religieuses, autant de crises qui contraignent à la remise en question. L'État est lui aussi repensé, dans la diversité de ses modalités, mais à partir de paramètres renouvellés : le réalisme politique de $M$ achiavel pense l'homme tel qu'il est, et expose les principes de son insertion concrète dans les mécanismes divers des pouvoirs terrestres, tandis que Thomas $\mathrm{M}$ ore, dans son Utopie, examine de manière critique l'organisation des rapports sociaux et la vie politique de son époque, et propose, par le moyen de la comparaison littéraire, un modèle politique et social nouveau et subversif.

Cette première partie de l'ouvrage montre donc comment surgissent des points d'origine, des commencements, dans tous les domaines de la pensée, et il est fait une 


\section{4 - Philosophiques / Automne 2002}

part importante à la découverte et au développement de l'imprimerie, dont le rôle dynamique fut capital pour la diffusion de ces idées et la continuation de ces commencements.

La seconde partie examine la façon dont ces idées cheminent et se développent pendant le siècle de la raison (le XVII e), siècle des crises et des révolutions. Les auteurs y montrent comment se conquiert peu à peu la liberté dans tous les domaines, liberté civile et politique, liberté intellectuelle, cependant que se structure encore plus fermement l'ordre classique.

Les deux premiers chapitre s'attachent à rendre compte des événements politiques en A ngleterre, qui cherche son équilibre politique, entre royauté et parlementarisme, au milieu des tensions religieuses permanentes, qui mènent d'ailleurs à la scission de l'Église d'Angleterre.

Dans ce contexte de crise de l'État se développent plusieurs théories politiques fondamentales et fondatrices, ce qui justifie l'examen de la situation historico-politique anglaise au début de cette seconde partie. C'est le moment où l'on repense le droit et la loi, en particulier en tentant de comprendre les principes du droit, à l'état de nature et dans le cadre d'une communauté civile. Les auteurs examinent les positions contractualistes de Hobbes, de Locke. II s'agit pour ces penseurs de déterminer ce qu'est l'ordre social, pour construire un ordre politique juste et assurant la liberté de chacun. Pour Hobbes, Le Léviathan, pouvoir absolu et non rapporté à une transcendance, apparaît de fait comme une solution aux guerres civiles et aux controverses religieuses, en ce qu'il permet de structurer un nouvel ordre social fondé sur le pacte civil, qui requiert le consentement de ceux qui sont soumis au pouvoir politique. C'est également la notion de pacte social qui est pour Locke le fondement de la communauté civile, dans laquelle le pouvoir politique constitue un remède contre l'individualisme qui règne à l'état de nature.

Les chapitres suivants dressent les portraits des figures fondamentale de ce siècle, en suivant à peu près l'ordre chronologique, mais c'est dans cette succession que le lecteur reste parfois quelque peu surpris des sauts qu'on lui fait faire. Certes, l'ouvrage se présente plus, on l'a dit, comme un livre ressource, que le lecteur peut parcourir de manière non linéaire, mais dans la continuité des chapitres, et c'est la limite d'un tel ouvrage qui a pour objet de dresser le tableau le plus complet possible de l'ensemble des grandes problématiques de ces siècles, on souhaiterait peut-être que l'ordre ne soit pas uniquement temporel. Le saut d'un domaine à l'autre, de la philosophie politique aux progrès de la science, ou aux œuvres de D escartes et de Spinoza, est parfois un peu abrupt. En revanche, l'examen des œuvres intellectuelles ne se fait pas sans un exposé clair du contexte de leur apparition, et donne lieu à des synthèses très dynamiques, qui manifestent de la part des auteurs un désir de tenir sur les auteurs classiques un vrai discours, en place des clichés ou des résumés maintes fois rencontrés dans les manuels ou les livres de vulgarisation.

L'état et l'évolution des sciences de ce siècle sont traités dans deux chapitres, l'un sur Galilée, l'autre sur N ewton. De l'un et de l'autre, il est montré - ce qui justifie leur examen singulier - en quoi ils constituent une «révolution» scientifique, et les auteurs mettent l'accent sur leurs parcours intellectuels, les modalités de leurs découvertes, tout en exposant brièvement la teneur de leurs positions scientifiques. O n voit bien comment en ce siècle de la raison, la nouvelle conception du monde développée à la Renaissance, conduit d'une part à privilégier à la fois une vision mécaniste du monde, d'autre part à accorder une importance de plus en plus capitale, épistémolo- 
giquement, aux notions d'observation et d'expérimentation comme instruments de validation de la théorie. À cette époque, la science reste encore liée à la religion, (malgré ses oppositions à l'Église instituée), dont elle se détachera progressivement au siècle suivant. $M$ ais l'idée commence à se faire jour que l'entendement humain, parce qu'il peut s'élever à une conception pure des lois nécessaires de l'univers, s'égale en nature, sinon en degré, à l'entendement divin.

Le chapitre sur Descartes est assez original à sa manière, en ce qu'il rompt avec les manières traditionnelles d'aborder l'œuvre de ce philosophe. L'accent est certes mis sur la notion de sujet, puisque D escartes constitue, commel'a montré H usserl, le point d'origine de la philosophie du sujet au sens moderne, mais on ne met pas l'accent sur la théorie de la connaissance. On nous montre bien plutôt comment cette dernière est subordonnée à la philosophie pratique (la morale) et à l'exigence de liberté, dont il est montré qu'elle se pose, avec D escartes, en absolu.

Les auteurs nous proposent également un tableau clair de la France pendant le règne de Louis XIV, dans un chapitre où sont expliquées dans leurs grandes lignes les fonctionnements d'une société désormais organisée autour du point focal constitué par la Cour et l'autorité absolue du Roi. On nous montre la coexistence d'un ordre classique régi par la rationalité (avec un arrêt sur Pascal et Port Royal, par exemple), et d'une pensée non-conformiste représentée particulièrement par le mouvement libertin.

Cette existence parallèle entre deux axes de pensée est résumée, d'une certaine manière, dans l'opposition théorique entre l'empirisme de Locke, et le rationalisme de Leibniz (chapitre 12), à propos de l'origine de nos connaissances et de nos idées. La thèse lockienne de l'origine sensible des idées et son opposition à Descartes sont exposées de manière relativement détaillée et claire, de même que les liens entre cette théorie génétique de la connaissance et ses répercussions sur la philosophie morale, qui ne peut plus être pensée de la même manière si on nie l'existence innée de principes moraux dans l'esprit humain. Face à Locke, mais en même temps, comme un moment de synthèse entre ce dernier et $D$ escartes, Leibniz soutient la thèse d'un innéisme virtuel. Selon lui, I'universal ité des idées rend nécessaire leur innéisme, mais leur complet dével oppement ne peut s'accomplir que dans le temps et l'expérience sensible. Leibniz retravaille la notion de puissance et rend compte du monde comme d'un système de substances dynamiques, système créé par Dieu en vertu du principe de raison suffisante et du principe d'harmonie, et à la différence de D escartes qui voyait dans la pensée la fonction essentielle de la substance, Leibniz met en place la difficile théorie de la monade (ici exposée brièvement) qui est dite représenter et exprimer l'univers. II était impossible, dans le cadre des chapitres assez courts, de présenter un exposé détaillé de ces théories complexes, mais les notions essentielles sont posées, offrant ainsi au lecteur une vision globale des systèmes des auteurs et des liens entre leurs différents points théoriques d'une part, et leur parcours et leur œuvre d'autre part.

La troisième partie enfin, traite du siècle des Lumières, en insistant particulièrement sur l'esprit de liberté qui souffle dans toute l'Europe, et s'étend sur le N ouveau $M$ onde. La raison s'y révèle dans sa force critique et son pouvoir judicatoire, et l'ensemble des chapitres semble nous amener progressivement vers ce qui est présenté comme le lieu de son épanouissement et son œuvre ultime, la R évolution française.

Dans un chapitre introductif, $M$ adame Boulad-Ayoub dresse le portrait rapide des révolutions intellectuelles, politiques et sociales de ce siècle, révolutions qui s'effectuent selon le principe énoncé par Kant : «Sapere aude, aie le courage de te servir de 


\section{6 - Philosophiques / Automne 2002}

ton propre entendement! », révolutions de la rationalité nouvelle qui, tout en reconnaissant leurs dettes à l'égard des méthodes et des enseignements des siècles précédents, travaillent à les dépasser pour faire souffler partout le vent de liberté qui envahit tous les domaines. On y voit régner l'esprit philosophique, défini par Diderot comme un «esprit d'observation et de justesse, qui rapporte tout à ses véritables principes», et la figure dominante est celle de l'honnête homme, «homme éclairé, pénétré d'esprit critique, pratique et soucieux de la réalité quotidienne». C'est aussi le siècle où s'épanouissent les salons, lieu de diffusion des Lumières et de lutte contre l'obscurantisme. Les intellectuels et les artistes mettent au cœur de leurs préoccupations le thème du bonheur, qui, s'il est recherché sous la direction de la raison et associé à la vertu, doit conduire à I'harmonie, aussi bien personnelle que collective. Puisque l'homme vit en société, il convient de penser précisément les principes et les lois qui régissent ces sociétés, afin de retrouver les fondements d'un agir à la fois rationnel et soucieux de la personne, de ses désirs et de ses intérêts fondamentaux, ce qui explique que les auteurs de ce siècle retravaillent la notion de nature, dans laquelle on situe le fondement de la liberté humaine, et qui est repensé comme une sorte de législateur universel.

Dans la continuité du retour à l'homme et à son intra-mondanéité, qui prend racine dans les siècles précédents, s'effectue dans cette période ce que nous pouvons interpréter comme une focalisation sur le point : on revient définitivement à l'homme comme point dans un espace désormais quadrillé et mesuré rationnellement grâce aux instruments méthodologiques nouveaux de la science, on revient à l'ici et maintenant de la sensation et de la perception, qui sont pour l'empirisme moderne de H ume ou le sensualisme de Condillac les points d'origine de toutes nos connaissances, on revient au moment singulier du plaisir, en particulier dans les arts, mais aussi dans les mœurs. Ce recentrage sur le point n'est cependant pas auto-limité : c'est à partir de ce retour à l'immanence que va se produire le passage, médiatisé de manière nouvelle, vers I'universel et le transcendant (voire le transcendantal). Le point est resitué dans l'espace infini, le moment dans le temps illimité, la nature morte, qui donneà voir l'objet dans sa plus pure immanence, est par là même ce qui semble le seul passage possible vers son éternité et son essence. II nous semble très intéressant, à la lecture de la synthèse des œuvres de ce siècle, de voir émerger ce qui ressemble finalement à l'émergence d'une conception dialectique du réel, qui accède à l'universel en faisant le passage par l'immanent, au lieu de penser le sensible et l'intelligible ou le transcendant comme deux modes auquel on accèderait de manière séparée.

On voit saillir dans ce contexte plusieurs personnalités dominantes : M ontesquieu, que ses analyses des règles sociales du droit politique instituent pour la postérité comme le fondateur de la science politique moderne; R ousseau, qui entreprend une anthropologie à travers laquelle il repense les concepts de nature, de droit, de loi, et de raison, et examine dans son Contrat Social la notion fondamentale de volonté générale, qui fait du peuple-souverain la source de la loi, conception qui sera au fondement même de la pensée de la révolution française; Voltaire, et ses combats contre l'intolérance et le fanatisme, qui font de lui le philosophe des lumières par excellence; Diderot, ainsi que tous les collaborateurs du projet emblématique des lumières, l'Encyclopédie, dont les auteurs font un exposé clair des objectifs, de la facture, des procédures et de la postérité. Plus tard G œthe, dont l'œuvre multiple traverse le siècle et peut être vue comme le miroir de ses évolutions intellectuelles et idéologiques. Enfin Kant, qui effectue ce qu'il nomme luimême sa révolution copernicienne dans le domaine de la théorie de la connaissance, en s'interrogeant non plus sur les objets de la connaissance eux-mêmes, mais sur les 
conditions de possibilité de cette connaissance. À travers un exposé succinct de sa philosophie transcendentale, qui réattribue à la raison sa fonction à la fois critique et législatrice, les auteurs nous donnent la mesure de l'ampleur de sa contribution à la philosophie moderne. On regrettera cependant que l'accent soit mis uniquement sur la philosophie spéculative, et qu'il ne soit pas fait état du pan essentiel de son œuvre qu'est sa philosophie pratique.

L'ouvrage se termine enfin avec un examen de ce qui est considéré par les auteurs comme l'aboutissement logique, la réalisation, au sens propre du terme, de ces grands mouvements de pensée et de libération, à savoir la refondation du corps social et politique qui s'effectue avec la révolution américaine et la déclaration d'indépendance des Etats-Unis d'une part, la Révolution française d'autre part. Les auteurs mettent l'accent, pour cette dernière, sur la rupture formidable qu'elle constitue pour les peuples, qui voient le vieux monde s'écrouler, tout en montrant que c'est bien l'ensemble des idées «qui ont animé la pensée politique des Lumières, c'est-à-dire la notion de loi, la notion de constitution, la notion de droits de l'homme», qui « vont se retrouver coulées dans la Déclaration des droits de l'homme et du citoyen ». L'avènement de l'état de droit et la fondation de la République, sur lesquels s'achève le long chemin que le lecteur vient de parcourir, ressemble effectivement au fruit Ionguement mûri dont le livre entier vient de nous montrer la croissance, à partir de ses germes éloignés et encore ignorés comme tels, dans l'éveil de la R enaissance.

Ce livre se présente donc à la fois comme une bonne vulgarisation et une invitation à l'approfondissement, une promenade dans laquelle l'érudit aura plaisir à retrouver des textes connus, des portraits chaleureux des auteurs qu'il apprécie, et des précisions sur d'autres dont il n'est pas spécialiste. L'ouvrage dans son ensemble fait bien le point sur les correspondances entre les divers domaines de la pensée, en ce sens qu'il met l'accent sur l'évolution des états d'esprit à chaque époque, et fait également état des interactions permanentes entre les œuvres qui font changer les mentalités, et les mentalités qui modifient les œuvres, ce qui permet de comprendre plus précisément l'émergence des différentes productions de l'esprit.

Pour l'étudiant, auquel il s'adresse plus particulièrement, ce livre représente une excellente ressource, puisqu'on y trouve les références aux figures incontournables, des textes canoniques, et une bibliographie riche. Les chapitres sur les auteurs sont clairs, les œuvres majeures sont au minimum citées, si elles ne sont pas traitées spécifiquement. Le cadre de ce travail ne permettait pas aux auteurs de rentrer dans le détail de chaque œuvre, de chaque auteur ou problématique, mais l'ensemble constitue une bonne vulgarisation, dans le sens le plus positif du terme, qui éveille chez le lecteur le désir d'en connaître plus sur les figures rencontrées, et lui fournit les moyens de le faire.

On regrettera cependant deux choses : la première tient à la conjonction entre un propos très vaste (plus de trois siècles d'histoire intellectuelle, politique et sociale) et à la composition collective de l'ouvrage, qui lui donnent parfois un caractère un peu fragmentaire, dont on comprend finalement qu'il n'était qu'une illusion, puisque la synthèse a eu lieu, et que la logique du chemin nous apparaît assez clairement, mais qui en cours de lecture déroute parfois, en dépit de la continuité chronologique qui maintient malgré tout une ligne directrice. Les chapitres sont thématiques, mais l'ordre des thèmes n'est pas toujours visible. Également, même si nous comprenons parfaitement qu'il était nécessaire de procéder à des choix dans les sujets et les auteurs traités, on aurait souhaité quelquefois une justification plus claire de ces choix. On trouve 
ainsi des chapitres entiers consacrés à la peinture et l'architecture, quand la musique est à peine évoquée.

Le second point tient à la forme du discours tenu. Ce texte s'adresse prioritairement à des étudiants, ou des esprits curieux qui n'ont pas nécessairement une formation académique, même s'il est aussi plaisant pour des spécialistes, pour les raisons que nous avons déjà évoquées. $M$ ais il entre dans cet ouvrage, en quelques endroits, une certaine irrégularité quant au niveau de discours, en ce sens que certaines notions complexes, ou simplement un peu spécialisées, sont parfois involontairement tenues pour évidentes, bien qu'il soit presque impossible que le public auquel s'adresse le livre en ait connaissance, quand par ailleurs, d'autres points relativement connus sont exposés comme si on s'adressait à un lecteur ignorant.

Cela n'enlève cependant rien au charme de cet ouvrage, qui a le mérite, à notre avis, d'éveiller ou de réveiller le désir de retourner aux œuvres et aux auteurs essentiels, qui sont présentés avec tout le relief que leur confère leur grande profondeur, et que fait apparaître clairement leur mise en perspective dans l'histoire.

AXELLE MARTIN

UQAM 